\title{
Discovering the Characteristics of Mathematical Programs
}

\author{
John W. Chinneck \\ Systems and Computer Eng. \\ Carleton University \\ Ottawa, Canada
}




\section{Motivation}

- Complex models, large scale:

- Unexpected results, bad performance, solver failure...

- Limited information returned by (e.g. NLP) solvers:

- Feasible, KKT conditions satisfied

- No improvement in many iterations: stopping.

- Unable to find feasible point.

- Too many iterations.

- Various specific failure messages...

- Questions:

- Why do I have this problem?

- How do I make the solver run better on this model?

- Needed: tools to discover the characteristics of models 


\section{Model Characteristics}

Some characteristics (e.g. for NLPs):

- Shapes of the constraints and objective (convex, concave, both, almost linear, etc.)

- Shape of the feasible region (convex, non-convex)

- Redundancy of constraints

- Location of feasible region

Insights gained:

- Better understanding of outcomes and behaviour

- Functions that can be approximated (e.g. linear)

- Constraints that can be ignored

- Best type of solution algorithm to apply

- Good starting point 


\section{Outline}

Theory

- Sampling for Characteristic Discovery

- What you can discover

- Tightening Sampling Box for Better Accuracy

- Sampling in Convex Envelopes

- Hit and run methods

- Approximating the analytic centre

- Point-Oriented Analysis

Practice

- MProbe software 


\section{Box Sampling}

- Random line segments within variable bounds:

- Uniform distrn for endpoint 1

- Uniform distrn for endpoint 2

- Interior points at fixed positions on line segment (e.g. 3 equally spaced)

- Default settings:

- 500 line segments

- 3 interior points per line segment

- Info from pts, info from lines

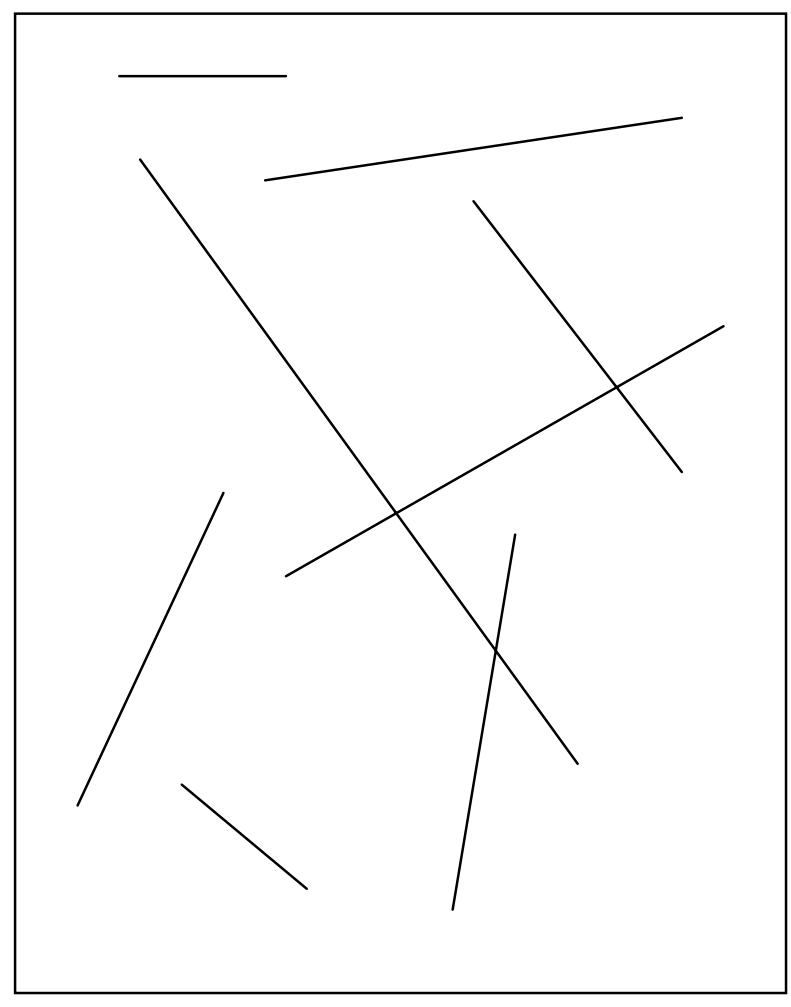




\section{Empirical Function Shape}

- Empirical Shape: based on sampled differences between actual and interpolated function values

- Depends on where you sample

- E.g. algebraically nonlinear function may be linear in region of interest

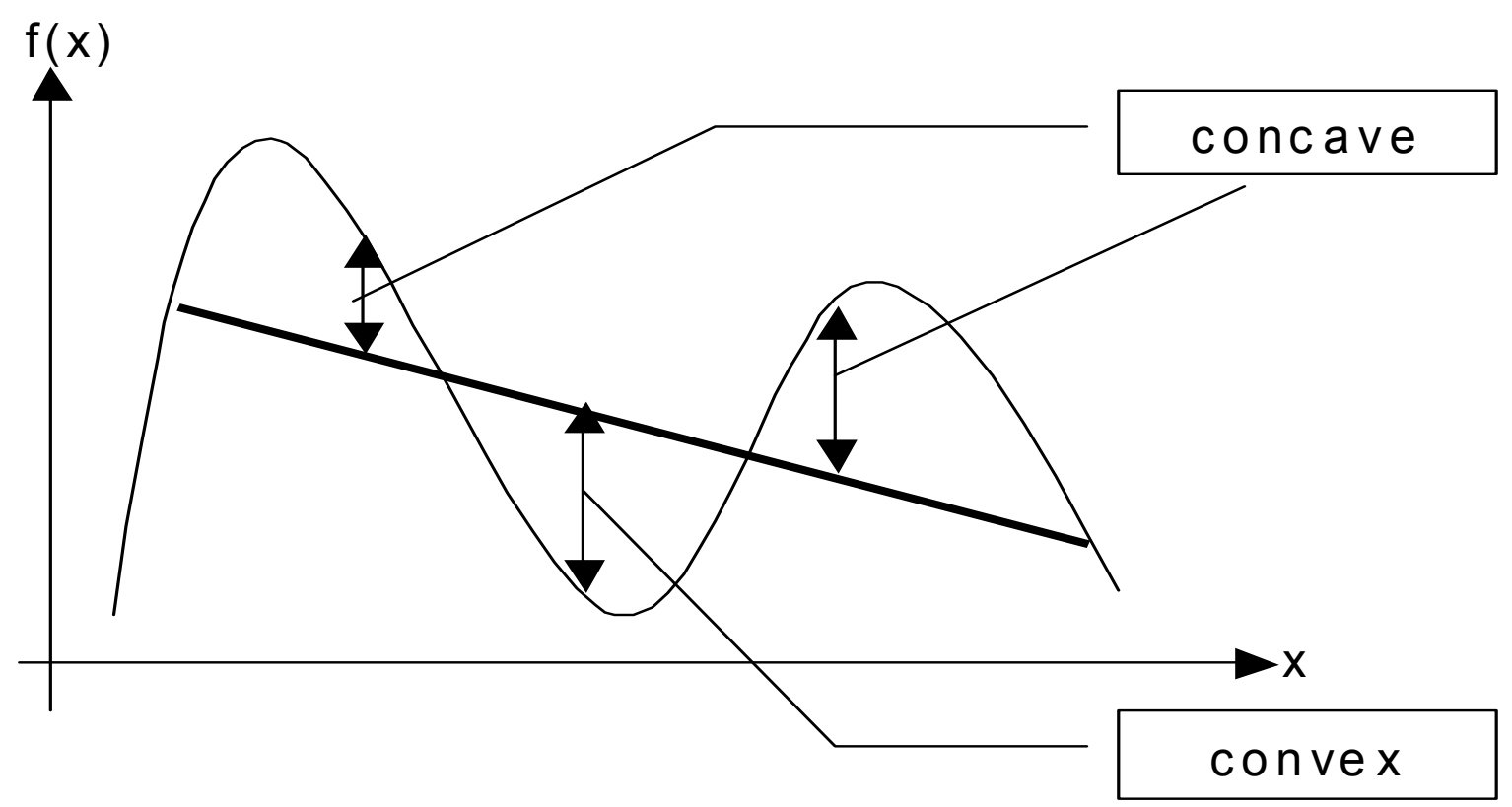




\section{Tolerances}

- Difference: interpolated - actual value

- Equality tolerance: if difference less than \pm tol $_{\text {equality }}$ then interpolated=actual at that point

- Almost tolerance: if difference is less than $\pm t_{0} l_{\text {almost }}$ then interpolated almost equals actual at that point

- E.g.: tol equality $=0.00001$, tol $_{\text {almost }}=0.001$

- Almost identifies candidates for approximation 


\section{Shape Histogram}

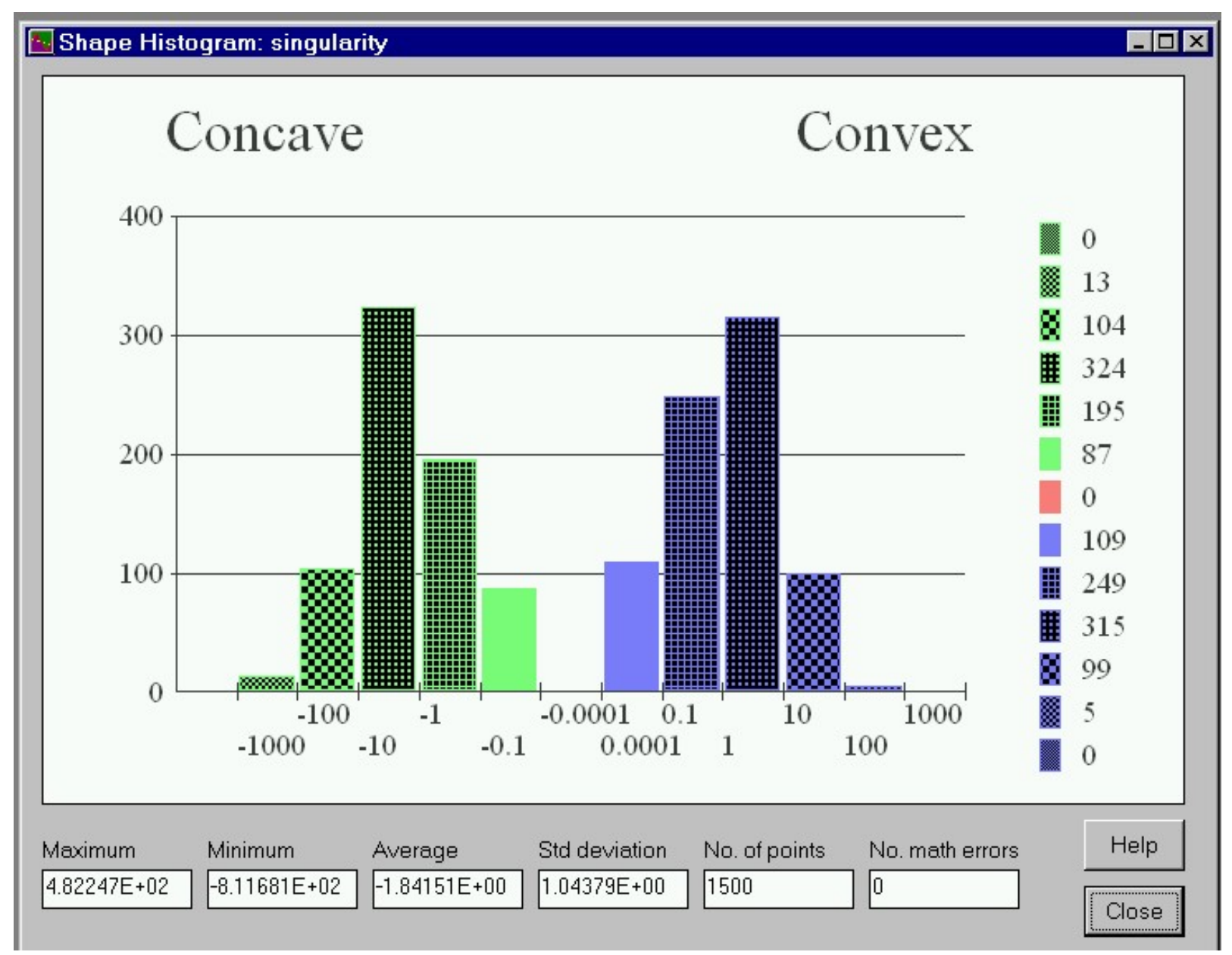

Discovering Characteristics of Math Programs 


\section{Function Shape Assessment}

linear: all diffs within \pm tol $_{\text {equality }}$

convex: all diffs are above -tol $\mathrm{equality}_{\text {and }}$ at least one above + tol $_{\text {almost }}$.

convex, almost linear: all diffs are above -tol equality, at least one is between + tol $_{\text {equality }}$ and + tol $_{\text {almost }}$, and none are above + tol $_{\text {almost }}$.

almost convex: at least one diff is between the -tol ${ }_{\text {almost }}$ and -tol $\mathrm{l}_{\text {equality, }}$ and at least one diff is above + tol $_{\text {almost }}$

concave: all diffs are below +tol equality, and at least one is below -tol $_{\text {almost }}$

concave, almost linear: all diffs are below +tol equality, $_{\text {, least one is }}$ between -tol ${ }_{\text {equality }}$ and -tol ${ }_{\text {almost }}$, and none are below -tol almost. .

almost concave: at least one diff is between +tol ${ }_{\text {almost }}$ and +tol equality, $_{\text {, }}$ and at least one diff is below -tol ${ }_{\text {almost }}$.

convex and concave: at least one diff is above + tol $_{\text {almost }}$, and at least one diff is below -tol ${ }_{\text {almost }}$.

convex and concave, almost linear: at least one diff is between

+ tol $_{\text {equality }}$ and the + tol $_{\text {almost }}$, and at least one diff is between -tol equality and -tol almost. 


\section{Summary of Constraint Shape Assessments}

\begin{tabular}{|c|c|c|c|c|}
\hline name & i.d. bound & Jalg. shape & Jemp. shape & reg. effect \\
\hline myquadratic & 0 Less than a constant & Quadratic & Convex & Convex \\
\hline mynonlinear & 1 Greater than a constant & General nonlinear, not quadratic & Convex and concave & Nonconvex \\
\hline myerrors & 2 Less than a constant & General nonlinear, not quadratic & Error & Shape errors \\
\hline singularity & 3 Less than a constant & General nonlinear, not quadratic & Convex and concave & Nonconvex \\
\hline sinquadratic & 4 Equality & General nonlinear, not quadratic & Convex & Nonconvex \\
\hline impossible & 5 Greater than a constant & Quadratic & Convex & Nonconvex \\
\hline ineffective & 6 Greater than a constant & Quadratic & Convex & Nonconvex \\
\hline ImpossEquality & 7 Equality & Quadratic & Convex and concave & Nonconvex \\
\hline mylinear & 8 Less than a constant & Linear & Linear & Convex \\
\hline multipleChoice & 9 Equality & Linear & Linear & Convex \\
\hline mixedTypes & 10 Less than a constant & Linear & Linear & Convex \\
\hline alllnts & 11 Interval & Linear & Linear & Convex \\
\hline
\end{tabular}




\section{(Un)certainty of Empirical Assessments}

- Linear report: confidence increases with testing

- Almost linear reports: confidence increases with testing:

- Convex, almost linear

- Concave, almost linear

- Convex and concave, almost linear

- Nonlinearity is certain if report is:

- Convex

- Concave

- Convex and concave

- xxx, almost linear

- Convex and concave is certain if report is:

- almost convex

- almost concave

- convex and concave 


\section{Constraint Effectiveness}

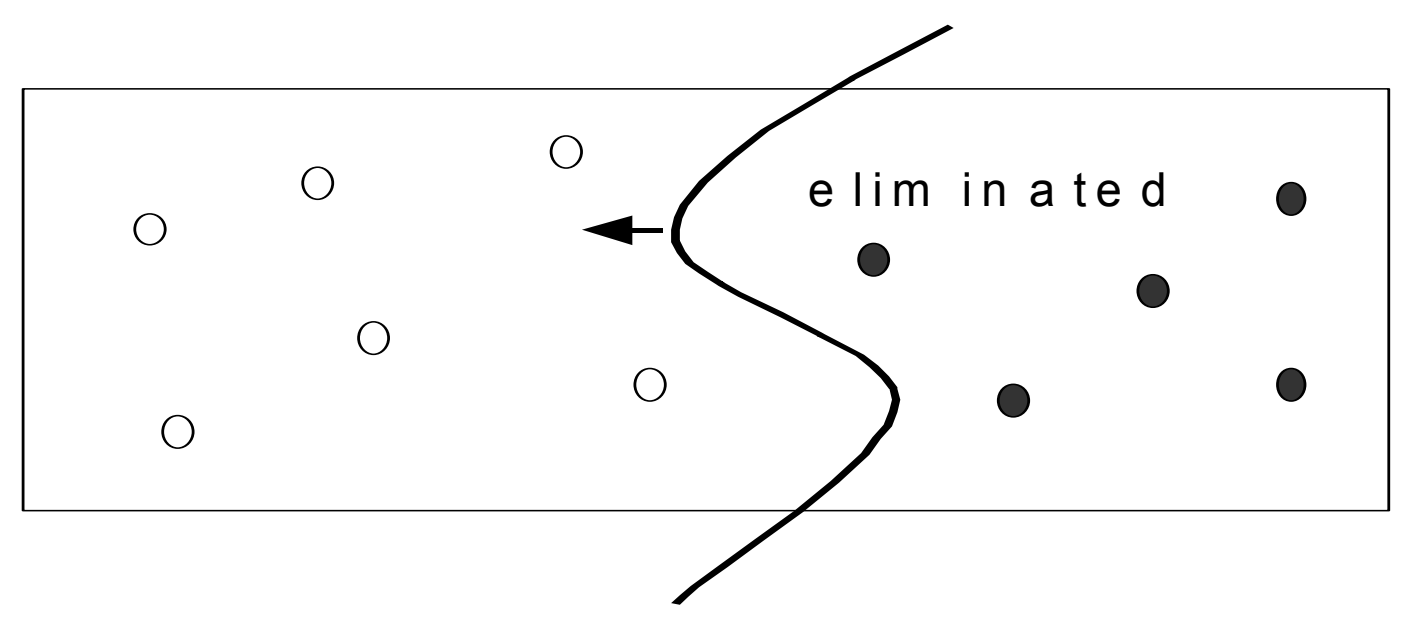

- Inequalities: what fraction of the sampling enclosure is eliminated?

- Equalities: fractions of sample points above, below, on the function? 


\section{Simple Feasibility \& Redundancy}

- Simple constraint

redundancy $(0 \%$

effective)

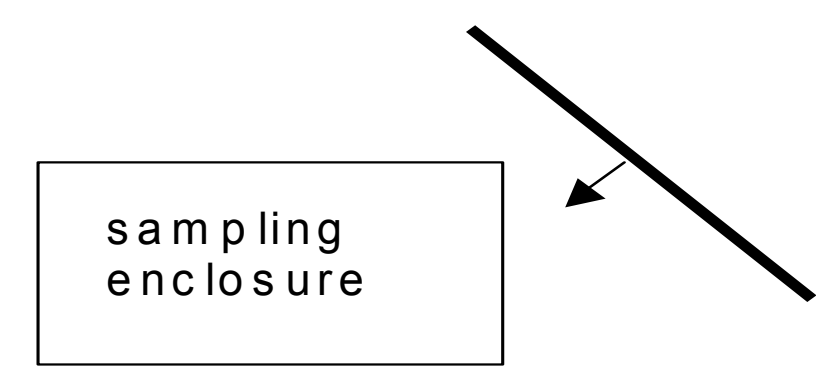

- Simple feasibility test

(100\% effective)

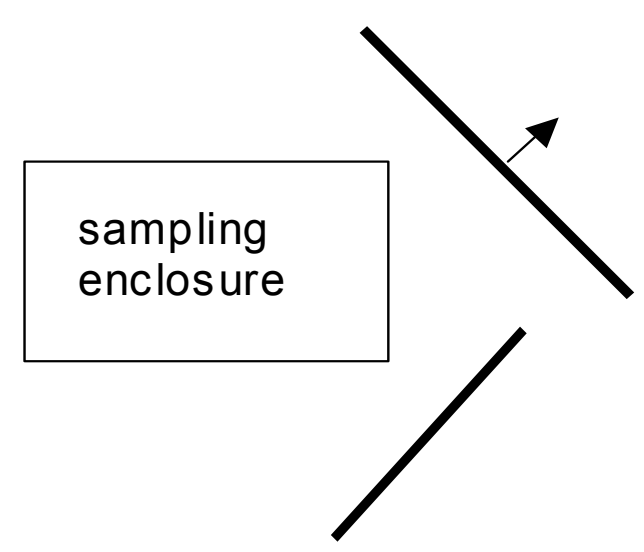




\section{Function "slope"}

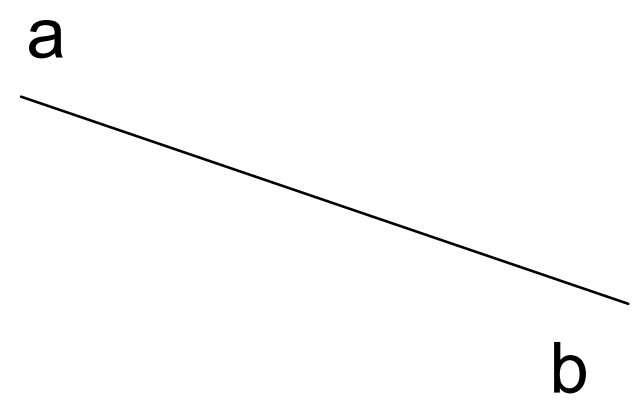

$|f(a)-f(b)| /($ length $a$ to $b)$

- Multidimensional idea of "steepness"

- Collect in histogram

- Especially useful for objective functions 


\title{
Objective Optimum Effects
}

- Objective sense and shape interact:

- global optimum possible by descent methods

- Local optimum likely

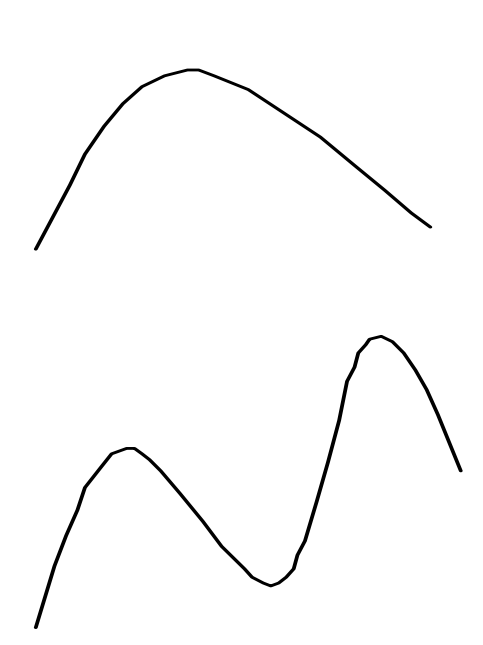

Max: glo bal opt possible M in: local opt like ly

\author{
Max or \\ Min: local \\ opt likely
}




\section{Constraint Region Effect}

- Effect on shape of constrained region

- Inequalities: sense

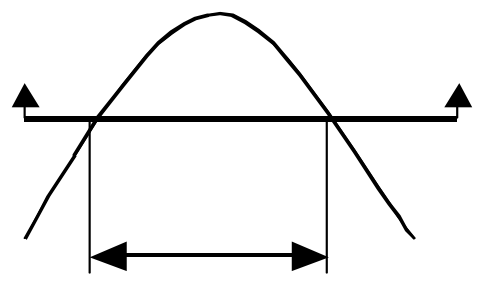

convex interacts with shape

- Equalities:

- Linear: convex region

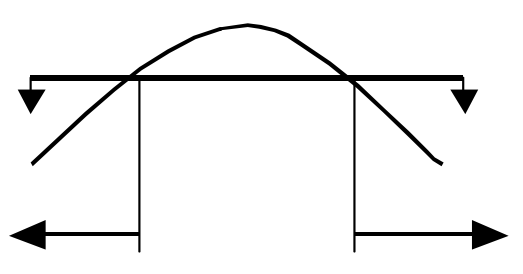
region effect effect

- Others: nonconvex region effect 


\section{Shape of Constrained Region}

- Assessing shape of the constrained region:

- All constraints have convex region effect: feasible region is convex (if it exists)

- Else constrained region is nonconvex

- Note: constraints sampled individually, results compiled
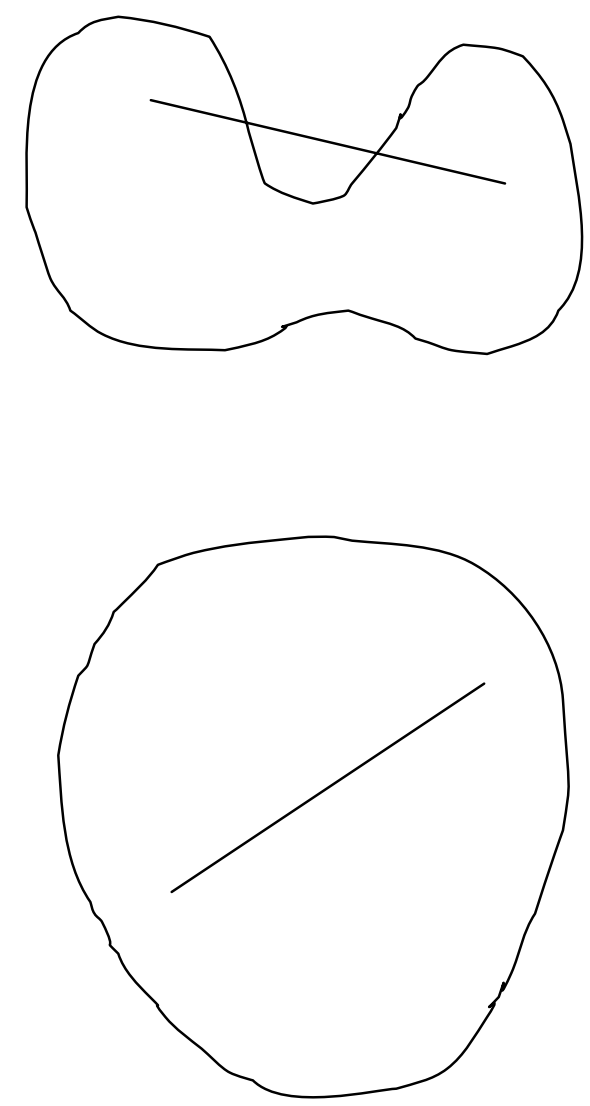


\section{Other Info from Sampling}

- Function value statistics

- histogram, max, min, etc.

- Objective function best sampled value and point (not nec. feasible)

- variables min and max sampled values

- Line segment length

- effect on conclusions 


\section{Tightening Sampling Box for Improved Accuracy}

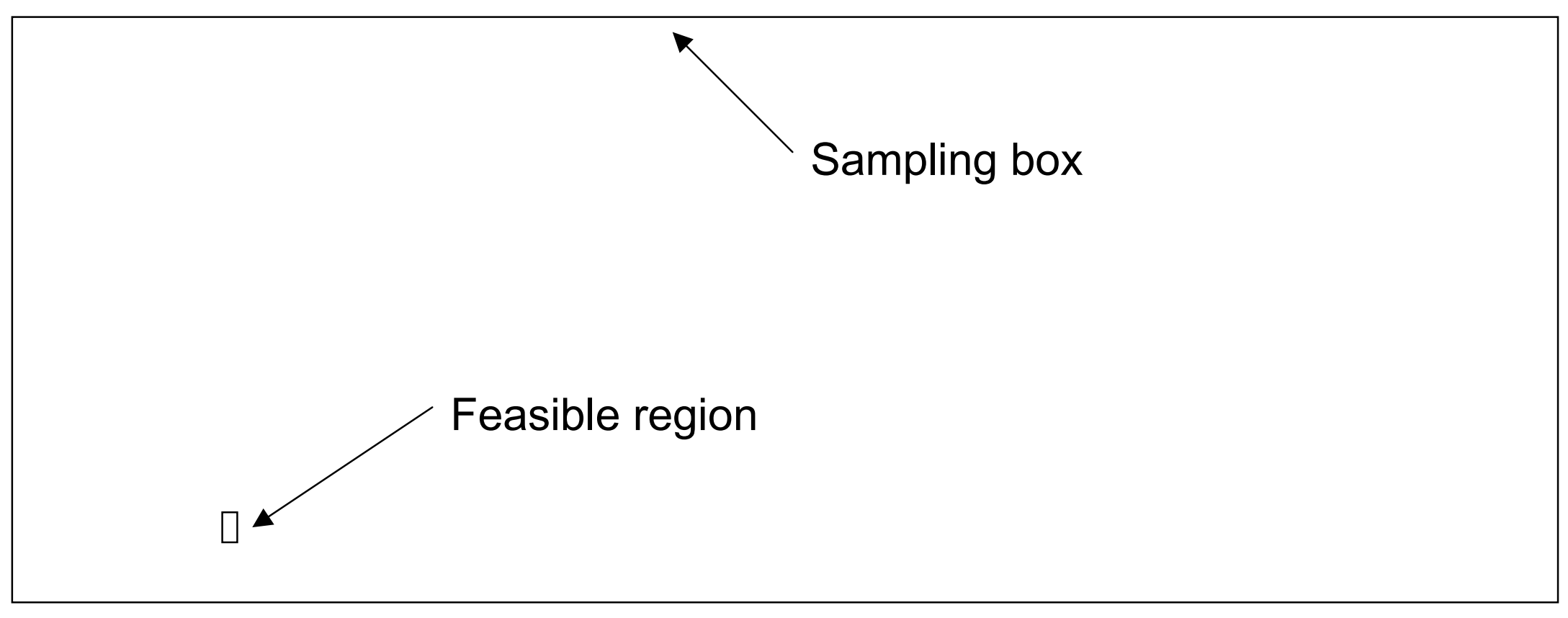

- Sampling box should be a close outer approximation of the feasible region 


\section{Tightening Bounds}

Methods:

- Manual adjustment

- Linear interval analysis

- Nonlinear interval sampling

- Get a nucleus box

- Range cutting

- Constraint Consensus bound tightening

- Max/min sampled values from convex enclosure (explanation deferred) 


\section{Linear Interval Analysis}

- Applies to the subset of linear constraints

- Like standard presolve: bound changes percolate

- E.g.:

- constraint $2 x_{1}-5 x_{2} \leq 10$ when $-10 \leq x_{1}, x_{2} \leq 10$

- Tighten $x_{2}$ lower bound by applying the constraint when $x_{1}$ is at it's lower bound: $2(-10)-5 x_{2} \leq 10 \Rightarrow$ $x_{2} \geq-6$.

- Conclusion: true bounds are $-6 \leq x_{2} \leq 10$. 


\section{Nonlinear Interval Sampling}

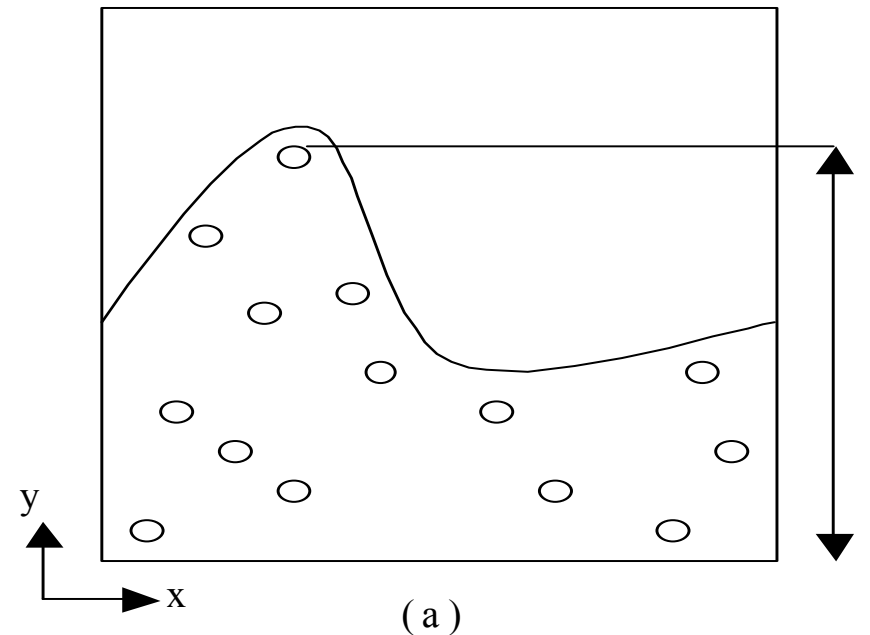

a) inequality

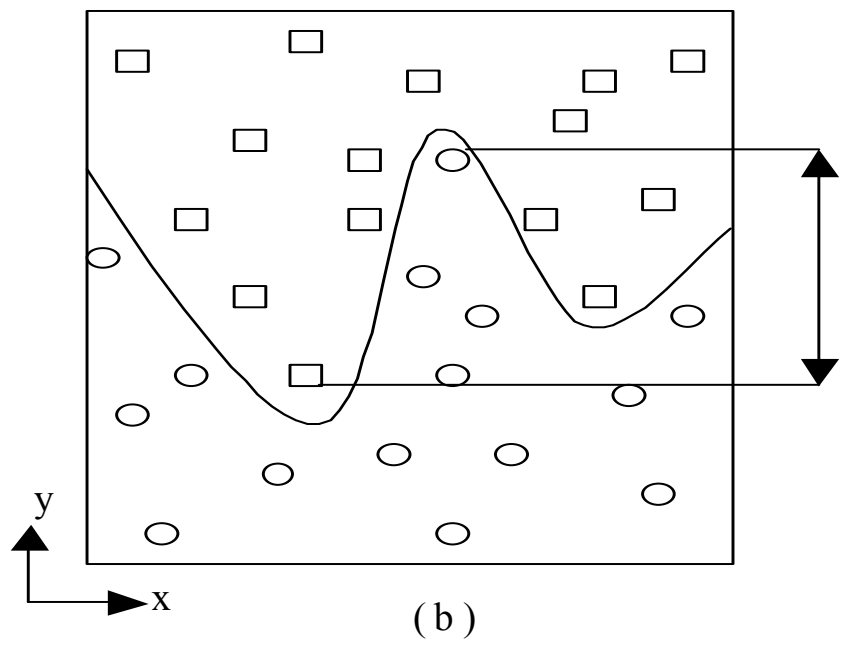

b) equality

- Apply to each constraint in turn

- overtightens

- non-overlap? return the gap itself 


\section{Get a Nucleus}

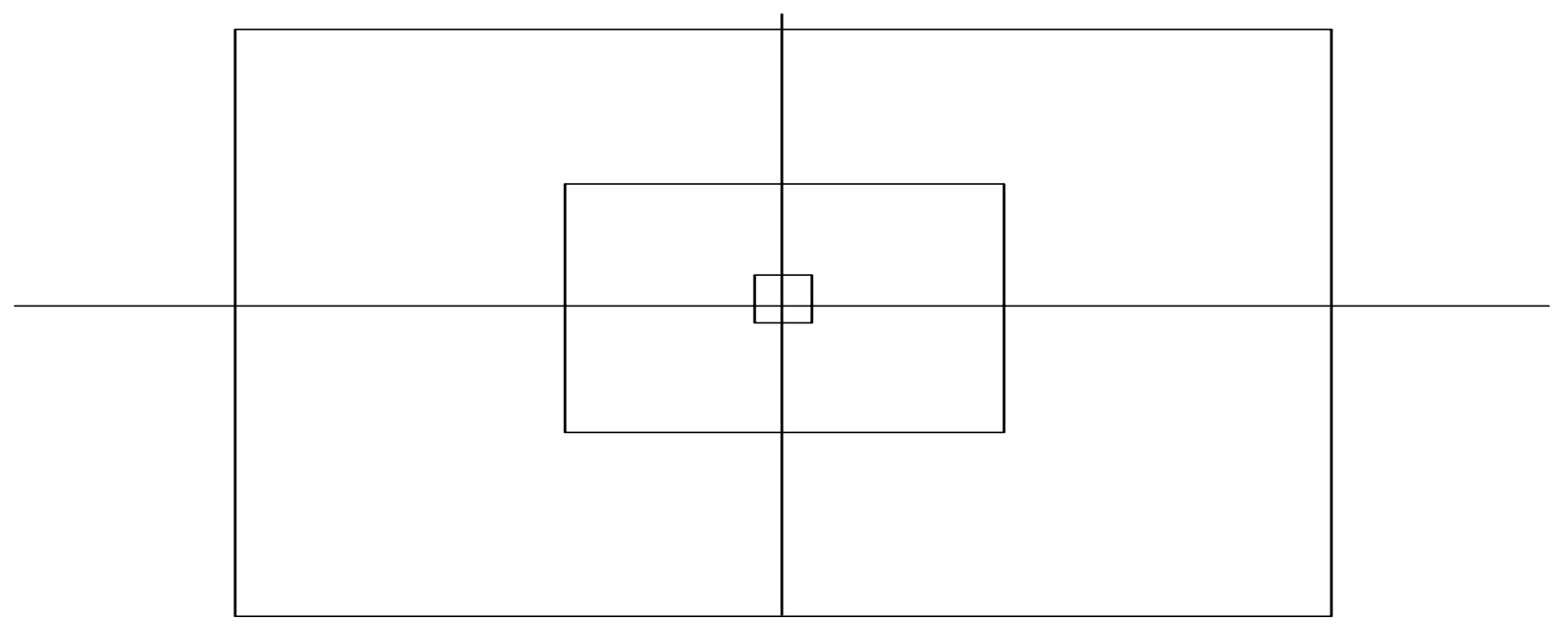

- For unbounded variables

- Look at constraints involving the variable that were never satisfied during interval sampling

- try gradually larger boxes centred at origin. Stop when next box shows no feasible points 


\section{Nonlinear Range Cutting}

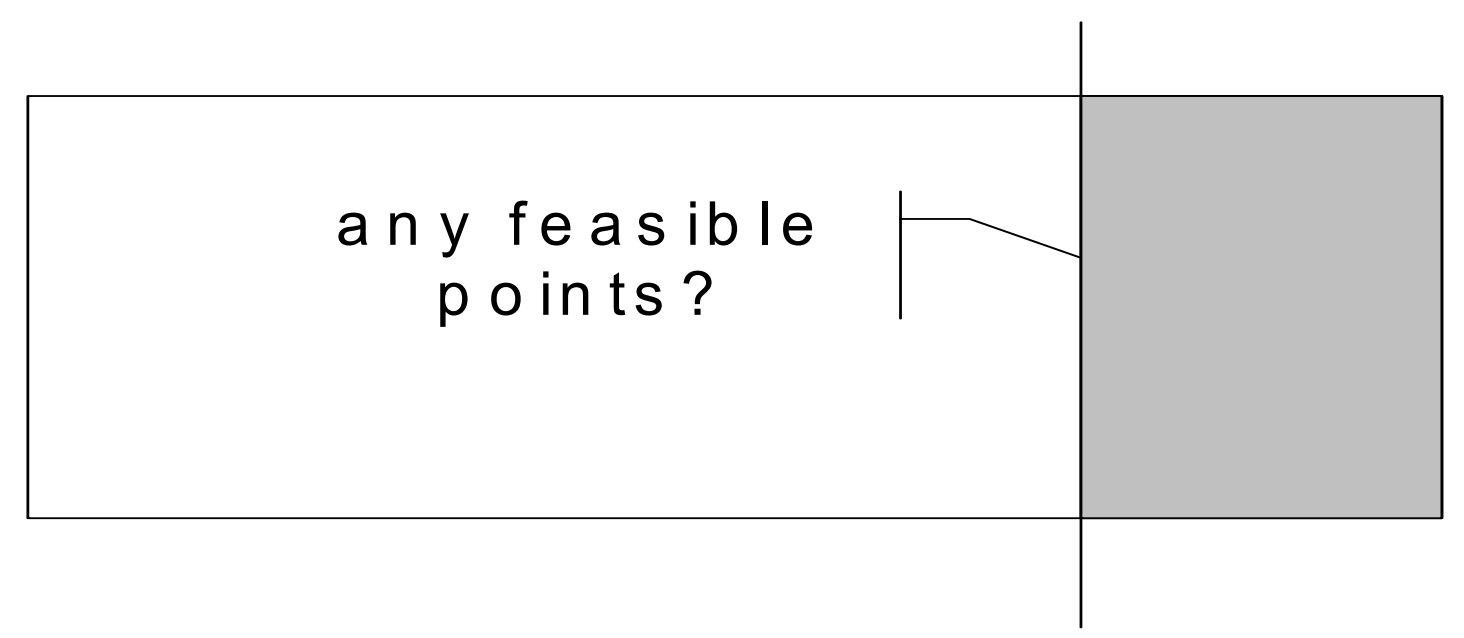

- Accept cut if at least one constraint never satisfied when sampling in the zone

- equalities: "satisfied" if find one pt $\leq$ rhs and one pt $\geq$ rhs 


\section{Constraint Consensus Heuristic}

- Quickly achieves approximate feasibility

- For each violated constraint: estimate vector to achieve feasibility

- Consensus vector: Component-wise average of feasibility vectors

- Update point using consensus vector

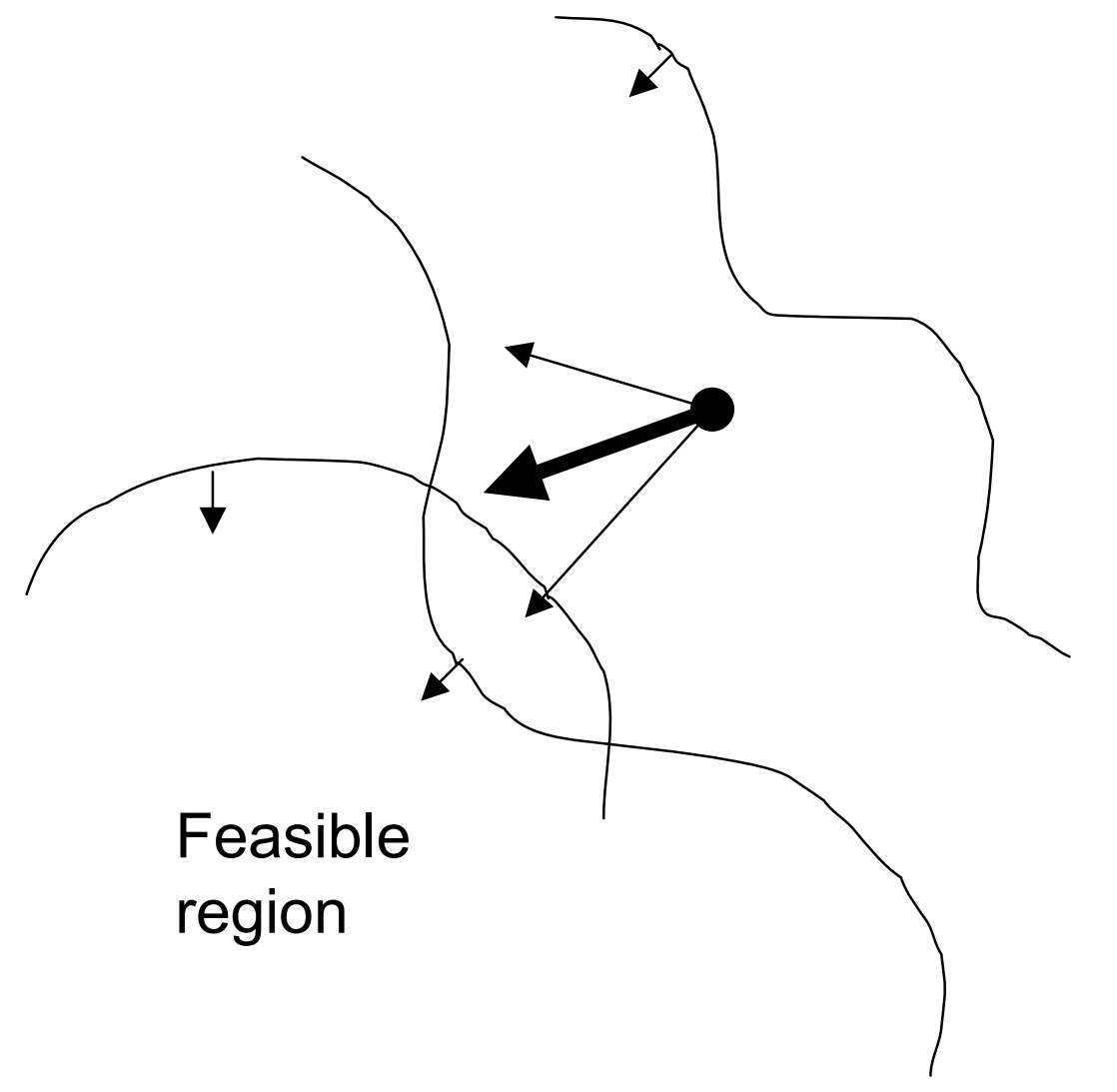

- Repeat until close to feasible 


\section{Constraint Consensus bound}

\section{tightening}

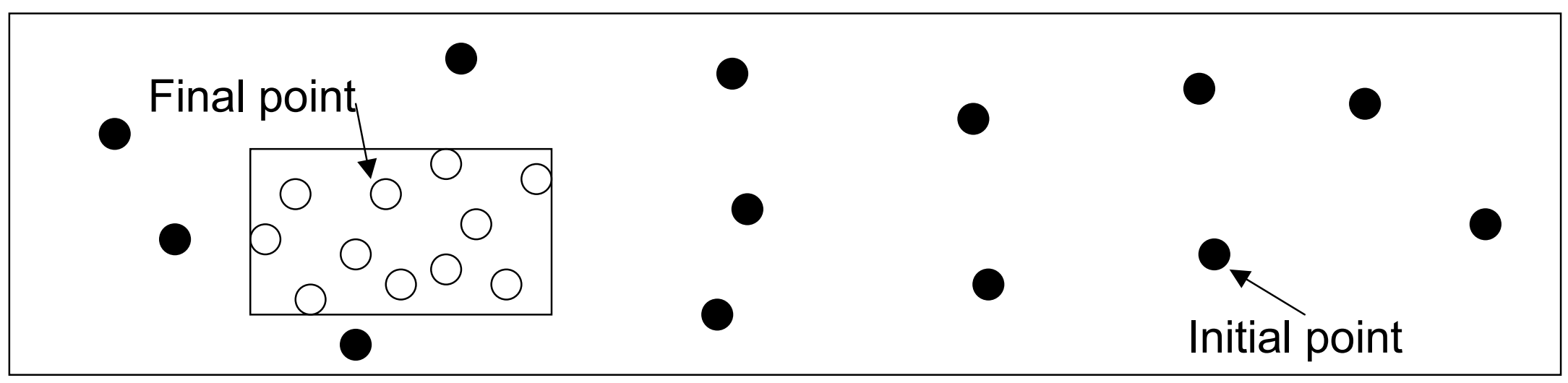

- Apply CC method from numerous random initial points in current sampling box

- Shrink bounds to encompass cloud of final points 


\section{Sampling in Convex Enclosures}

How do you find a convex region that encloses the feasible region?

Procedure:

1. analyze constraint region effects by box sampling

2. select inequalities that have convex region effects and all variable bounds

Sample via hit-and-run methods 


\section{Hit-and-Run Sampling}

- hit constraints are necessary; unhit constraints are redundant (relative to enclosure)

- estimate fraction of enclosure surface area

- non-enclosure constraints sampled as usual (shape, effectiveness, etc.)

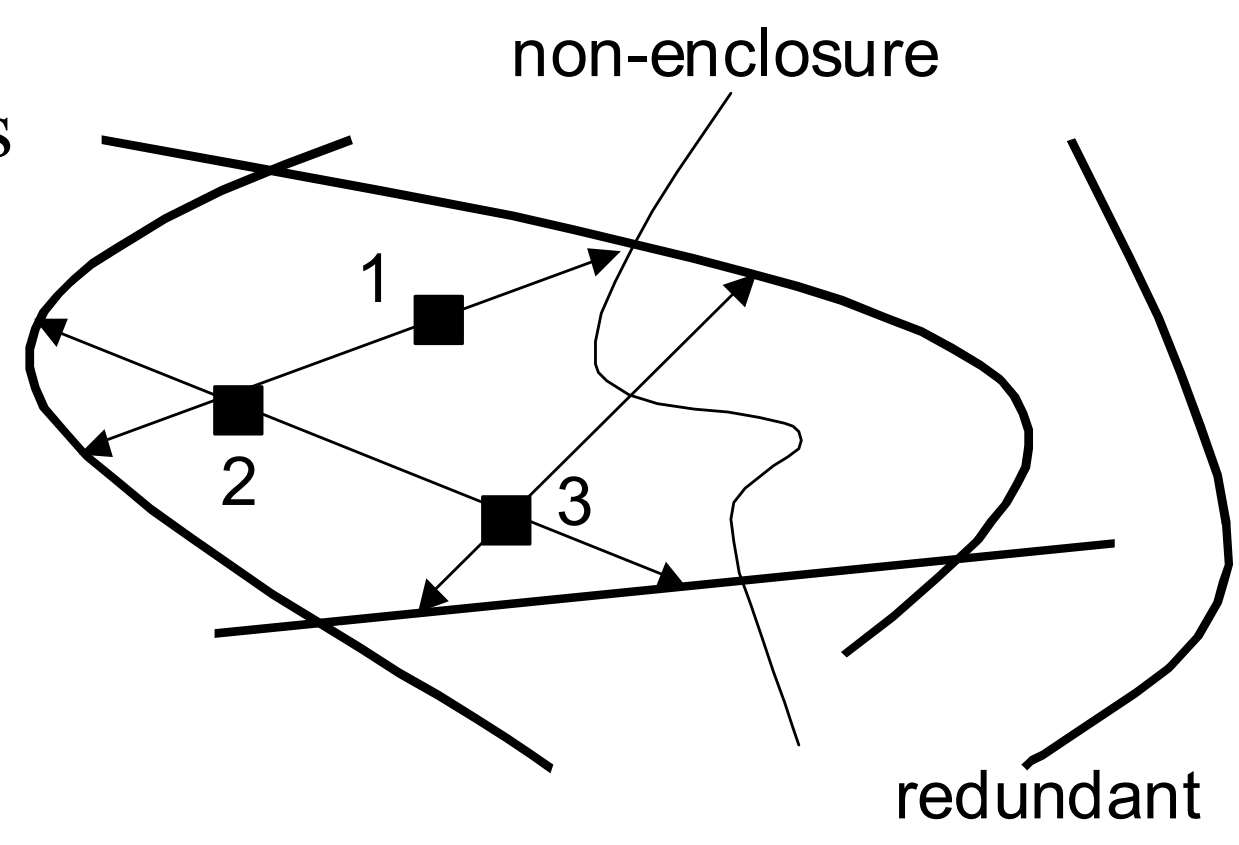




\section{Finding an Initial Feasible Point}

Need an initial feasible point to start hit-and-run

Method 1:

- Sample randomly until at least one constraint satisfied

- Thereafter use hit-and-run to keep constraints satisfied

- Stop when all constraints satisfied.

- Note: bias hit-and-run sampling rays according to variable bounds (long thin boxes are a problem)

Method 2:

- Apply constraint consensus method 


\section{The Analytic Centre}

Analytic centre: $\boldsymbol{P}$ is point that maximizes $W=\sum[\ln$ (distance to constraint) over all constraints.

- Best place to launch rays for estimating surface frac. Heuristic for finding analytic centre:

- Initial feasible pt is first estimate of $\boldsymbol{P}$. $W$ always calculated over necessary constraints found so far.

- Launch hitting rays from $\boldsymbol{P}$.

- Get new hit-and-run launch point $\boldsymbol{x}$.

- If $W(\boldsymbol{x})>W(\boldsymbol{P})$ then $\boldsymbol{P}=\boldsymbol{x}$.

Advantages:

- Pushes $\boldsymbol{P}$ away from discovered necessary constraints towards undiscovered necessary constraints

- Quick convergence to analytic centre. 
"replace current bounds with max/min sampled values"

- After convex enclosure sampling: tighten bounds

- Use hit points to (over)tighten the variable bounds

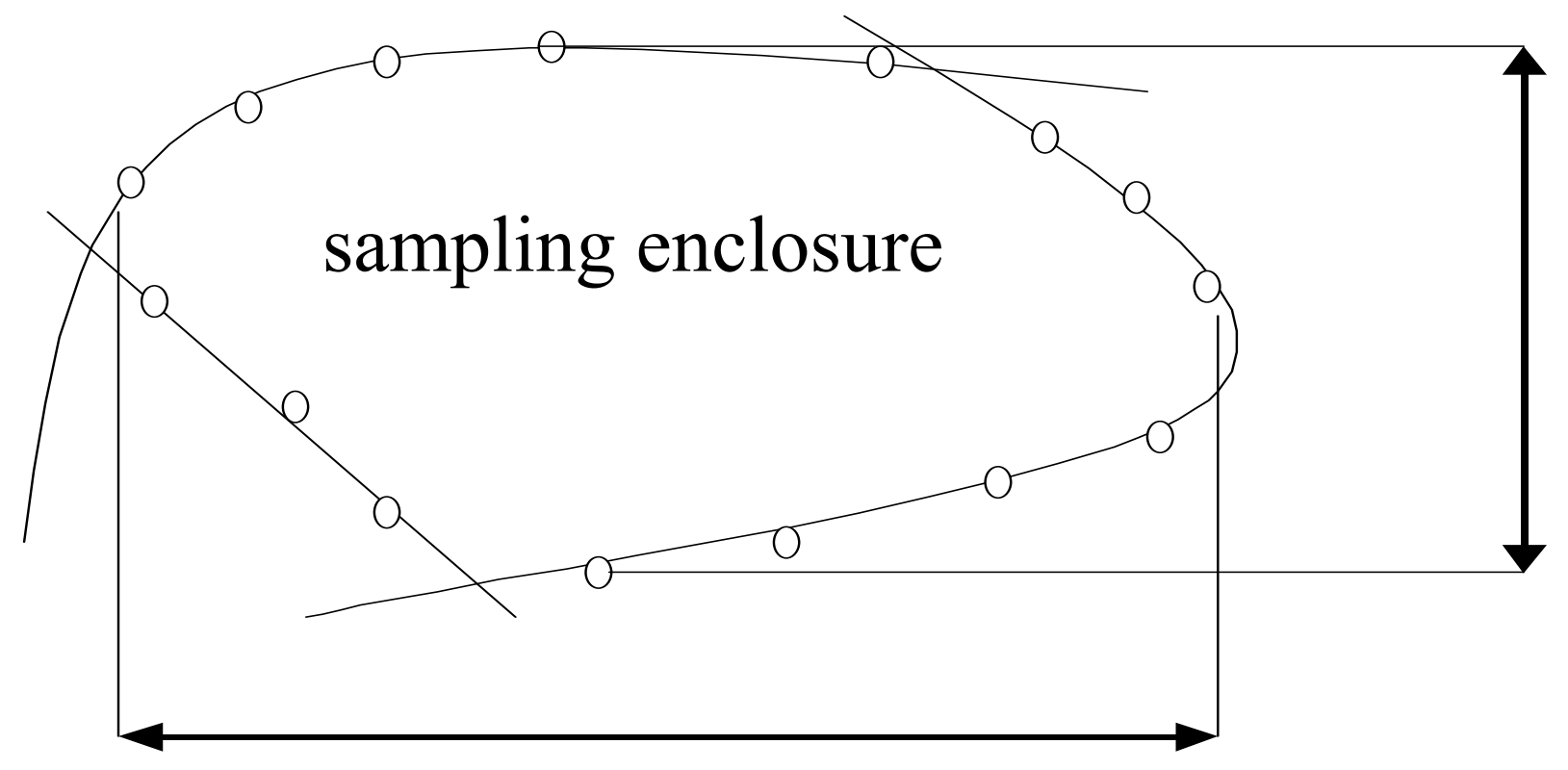




\section{Point-Oriented Analysis}

- Finding (near) feasible points

- What is a good starting point?

- Finding (near) optimal points

- Analyzing features of points

- Why did my solver stop here? 


\section{Finding (Near) Feasible and Optimum Points}

- Finding a near-feasible point:

- constraint consensus method

- Finding a near-optimal point:

- keep track of feasibility status and objective function value of all sampled points.

- Note best found point and best found feasible point 


\section{Analyzing Point Features}

- Create a small box around a point:

- E.g. shrink bounds to $1 \%$ of each current edge dimension, centred around point

- Look at objective "flatness" in the box

- Histogram of objective "slope" in the box.

Flat (1-D)
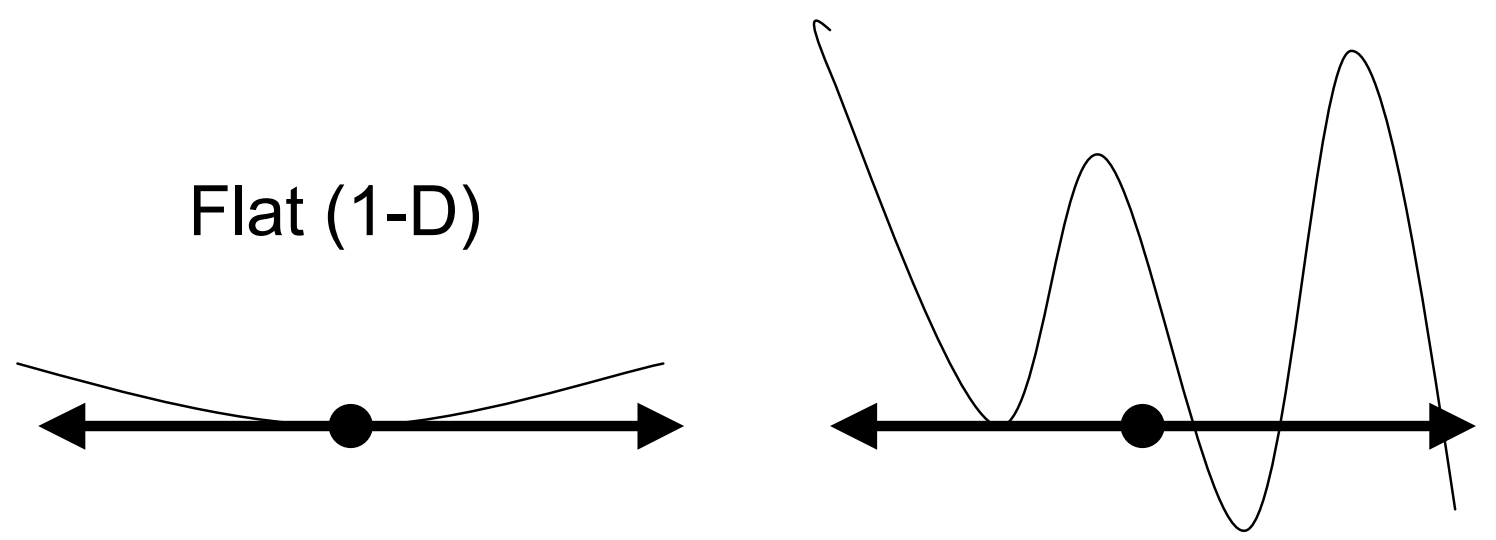


\section{MProbe}

- Software tool embodying these and other analytic methods

- Reads AMPL, GAMS, MPS files

- Essential part of an integrated development environment for math programming 


\section{MProbe Workshops}

\section{Variables Workshop}

- Shift, tighten variable bounds

Constraints and Objectives Workshops

- Analyze shape, effectiveness, redundancy, set up convex enclosures for tighter sampling

Constrained Region Workshop

- Analyze shape of feasible region

Points Workshop

- Exchange points with solvers, look for near-feasible points, etc. 


\section{Additional Features}

- Model statistics

- "Snapping" of integer/binary variables

- Spreadsheet-like displays of constraints, objectives, variables

- View subsets by category (e.g. only nonlinear constraints, only binary variables)

- Sort on any column

- Navigate (e.g. see all constraints that contain variable $x)$

- User adjustment of tolerances, histogram cells

- Text file trace of session

- Help system 


\section{Constraints Workshop}

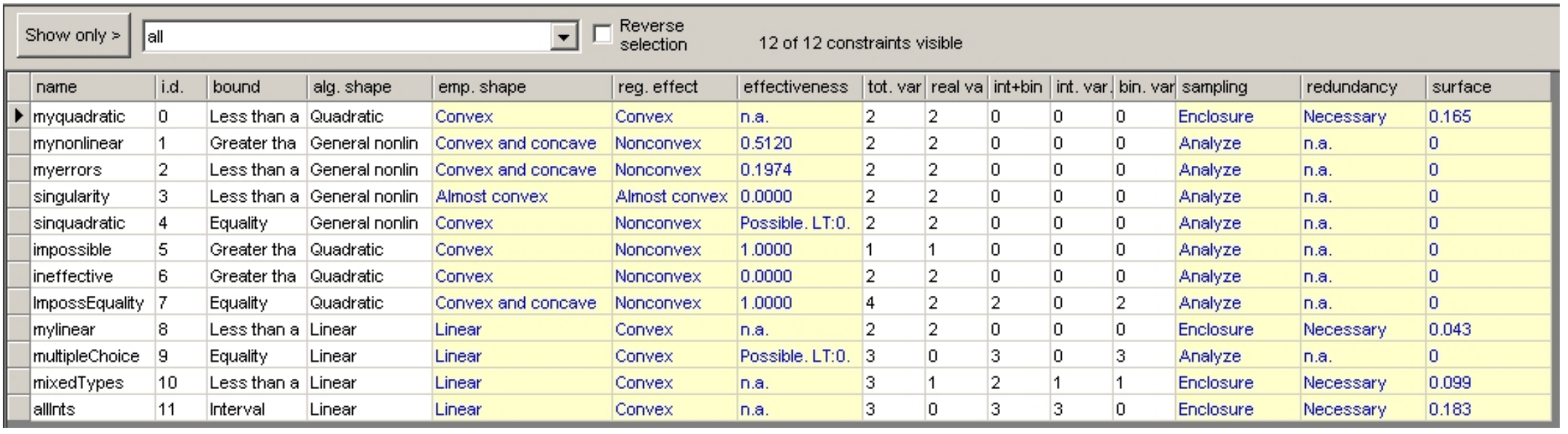




\section{Visualizing Shape: Function Profile}

- 2 dimensional plot between 2 endpoints in $\mathrm{n}$ space

- End-points selectable and configurable in multiple ways

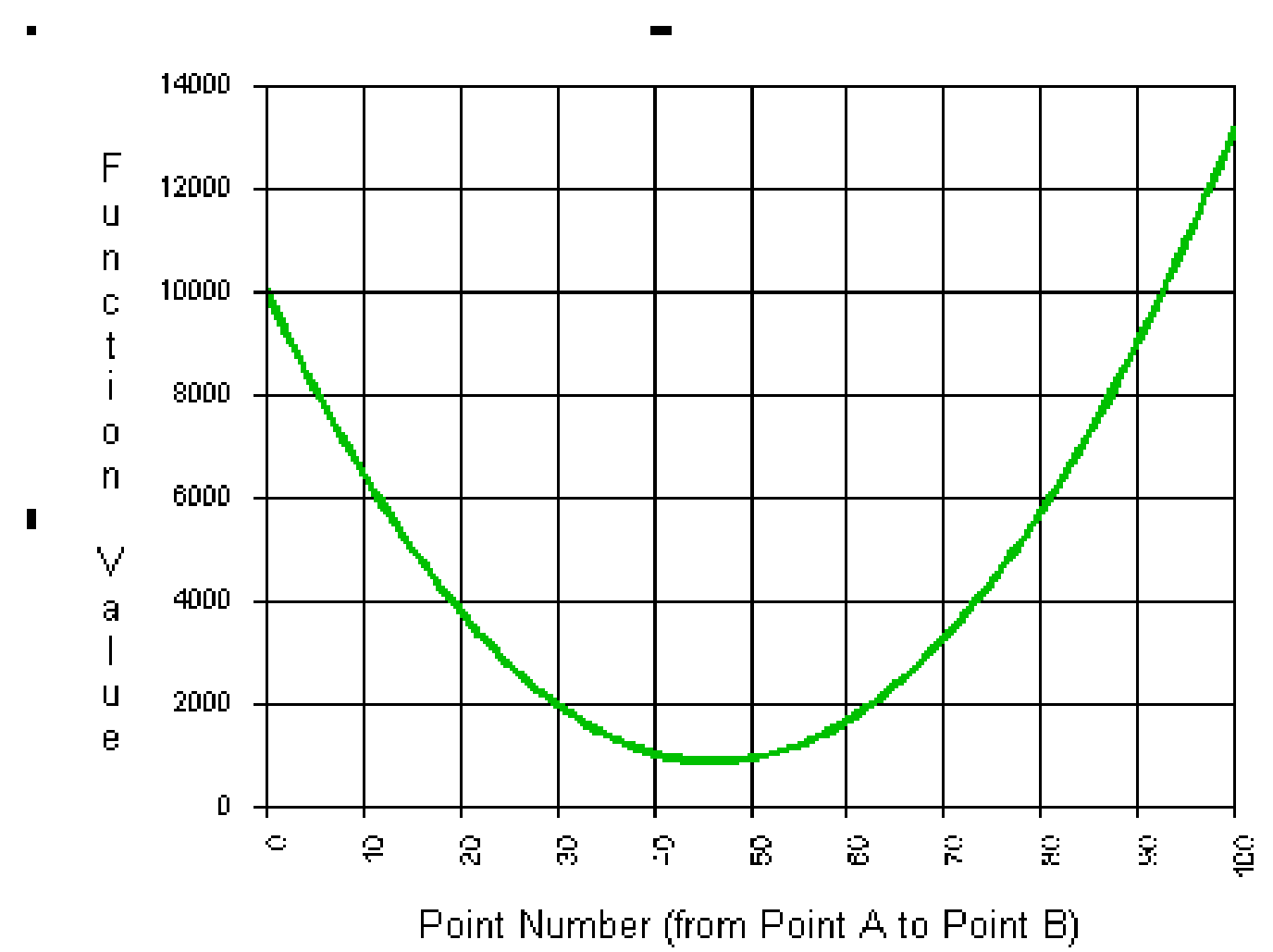




\section{Constrained Region Workshop}

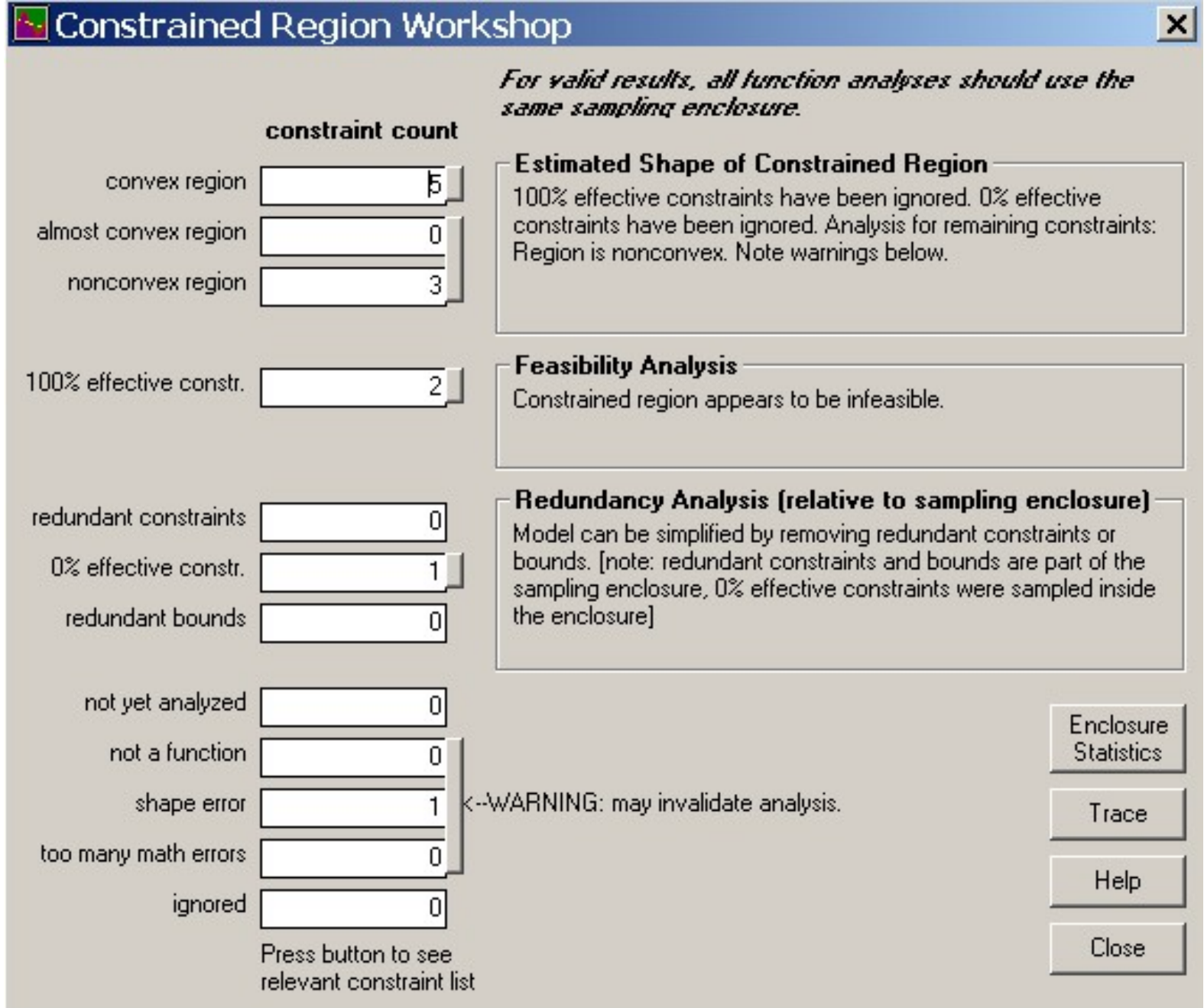




\section{Points Workshop}

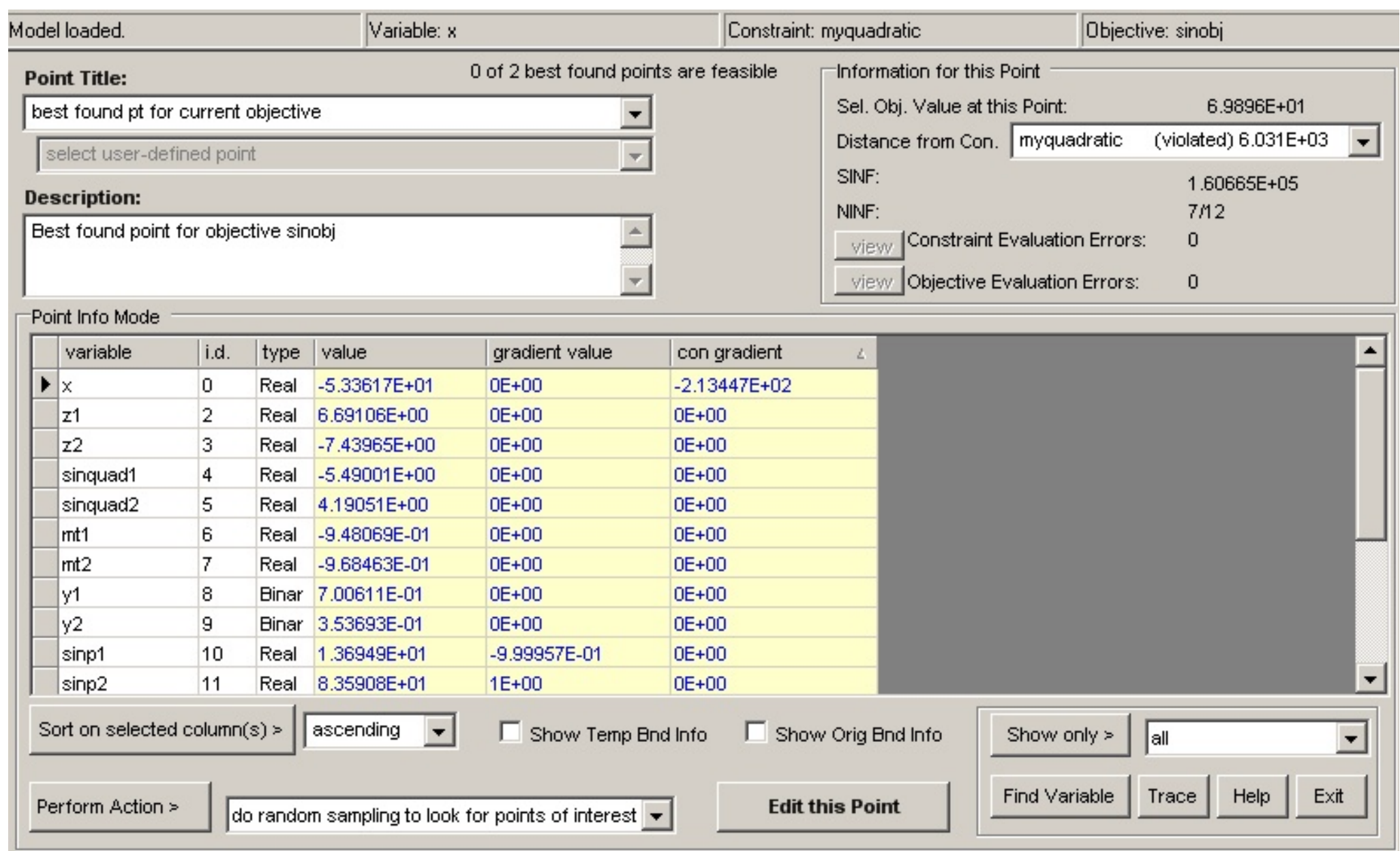




\section{Conclusions}

- Model probing and analysis tools a vital part of IDE for math programming

- Shape analysis tools are heuristic and based on random sampling

- Tools don't always work, but often do.

- Can be slow for very large or very complex models

- Performance depends on characteristics of the model

- Download: www.sce.carleton.ca/facultyl chinneck/mprobe.html 


\section{Research Directions}

- Better Sampling

- Interior pts on line segments means more samples towards centre of sampling box

- Better bound tightening

- Better interaction among the component methods

- Determining best approximations of functions (e.g. best piecewise linear approximation)

- Connection to computer algebra system (Matlab?)

- Tool for identifying implied equalities

- Etc........... 


\section{References}

- Chinneck, J.W. (2003), "The Constraint Consensus Method for Finding Approximately Feasible Points in Nonlinear Programs", INFORMS Journal on Computing, to appear.

- Chinneck, J.W. (2002), "Discovering the Characteristics of Mathematical Programs via Sampling", Optimization Methods and Software, vol. 17, no. 2, pp. 319-352.

- Chinneck, J.W. (2001), "Analyzing Mathematical Programs using MProbe", Annals of Operations Research, vol. 104, pp. 33-48. 